Article

\title{
The High-Order Harmonic Generation from Atom Driven by Co-Rotating Laser Pulses Composed of Fundamental Frequency and High Frequency
}

\author{
Yanzuo Chen ${ }^{1,2}$, Xuxu Shen ${ }^{1,2}$, Wendi Lan ${ }^{1,2}$, Suyu Li ${ }^{1,2}$, Fuming Guo ${ }^{1,2, *}$ and Yujun Yang ${ }^{1,2, *}$ \\ 1 Institute of Atomic and Molecular Physics, Jilin University, Changchun 130012, China; \\ chenyz1117@mails.jlu.edu.cn (Y.C.); shenxx18@mails.jlu.edu.cn (X.S.); lanwd20@mails.jlu.edu.cn (W.L.); \\ sylee@jlu.edu.cn (S.L.) \\ 2 Jilin Provincial Key Laboratory of Applied Atomic and Molecular Spectroscopy, Jilin University, \\ Changchun 130012, China \\ * Correspondence: guofm@jlu.edu.cn (F.G.); yangyj@jlu.edu.cn (Y.Y.)
}

check for updates

Citation: Chen, Y.; Shen, X.; Lan, W.; Li, S.; Guo, F.; Yang, Y. The High-Order Harmonic Generation from Atom Driven by Co-Rotating Laser Pulses Composed of Fundamental Frequency and High Frequency. Symmetry 2022, 14, 519. https://doi.org/10.3390/sym14030519

Academic Editors: Shicheng Jiang and Jigen Chen

Received: 30 January 2022 Accepted: 16 February 2022 Published: 3 March 2022

Publisher's Note: MDPI stays neutral with regard to jurisdictional claims in published maps and institutional affiliations.

Copyright: (C) 2022 by the authors Licensee MDPI, Basel, Switzerland. This article is an open access article distributed under the terms and conditions of the Creative Commons Attribution (CC BY) license (https:// creativecommons.org/licenses/by/ $4.0 /)$.

\begin{abstract}
By numerically solving the time-dependent Schrödinger equation (TDSE), the harmonic generation process of atoms irradiated by corotating laser pulses composed of a fundamentalfrequency and high-frequency field is systematically studied. Compared with the harmonic generated from atoms irradiated by counter-rotating two-color circularly polarized laser pulses, the harmonic efficiency of atoms irradiated by co-rotating two-color circularly polarized (CRTCCP) laser pulses with the same laser parameters is higher. The harmonics are generated by the multiphoton radiation transition after the bound electrons undergo a multiphoton absorption transition to a higher energy level. In addition, the variation of the harmonic efficiency with the field strength of different frequency components in the driving laser pulse is also studied. The circularly polarized harmonics with higher intensity can be obtained by optimizing the field strength of the driving laser field.
\end{abstract}

Keywords: circularly polarized high-order harmonic; co-rotating two-color circularly polarized laser field; selection rule; TDSE

\section{Introduction}

With the development of laser technology, the electric field intensity of laser pulses has reached the Coulomb field intensity that can be felt by electrons in atoms. Many nonlinear physical phenomena can be observed in atomic and molecular systems irradiated by such a strong laser pulse, such as high-order harmonic generation (HHG), high-order above-threshold ionization [1,2], non-sequential double ionization [3], etc. The coherent HHG from atoms irradiated by the strong lasers ranges from extreme ultraviolet to soft X-ray [4-12]. Therefore, HHG is widely applied in attosecond pulse generation [13-18], molecular structure imaging [19-21], high-frequency coherent light sources [11], etc.

The physical mechanism of HHG from atoms and molecules irradiated by linearly polarized lasers can be given by the three-step model. That is, driven by the laser pulse, electrons move away from the nuclear region. Then, as the direction of the driving laser field is changed, some of the ionized electrons have the opportunity to return to the parent ion and recombine with the ground state. Finally, the high-energy photon is emitted [22-24]. The polarization properties of HHG are closely related to those of the driving field. Generally, the HHG from atoms irradiated by linearly polarized lasers is still linearly polarized. The application of linearly polarized HHG is limited. The circularly polarized high-frequency coherent light sources are needed in some fields, such as the detection of magnetic materials [25] and the study of molecular symmetry [26-31].

Usually, circularly polarized HHG cannot be produced by the interaction of circularly polarized lasers with atoms. It can be attributed to the fact that when the driving field is circularly polarized, the direction of the ionized electron's velocity will change with time 
under the action of the laser electric field, which makes the electrons move far away from the parent ion, thereby reducing the opportunity of returning to the parent ion to generate radiation $[32,33]$. To obtain the circularly polarized harmonic laser sources, a scheme which uses a circularly polarized laser pulse composed of the fundamental frequency and its second harmonic with the opposite polarization direction to irradiate atoms and molecules is proposed [34-37]. By using this scheme, strong circularly polarized HHG can be observed. The harmonics at the frequency of times of the fundamental frequency are suppressed, and the adjacent integer-order harmonics show different circular polarization characteristics. On this basis, the ionization, photoelectron emission spectrum and nonsequential ionization process of the system under the action of this combined laser pulse were further studied.

The interaction between the counter-rotating two-color circularly polarized laser and the atom have attracted great attention. At the same time, researchers have also begun to study the interaction of the co-rotating two-color circularly polarized (CRTCCP) laser field with the atom. In 1995, Becker et al. [38] theoretically studied the HHG of atoms driven by the CRTCCP field, and found that under the action of this combined laser pulse, the intensity of the HHG is much lower than that obtained under the action of the counterrotating two-color circularly polarized laser field. In 2018, Busuladžić et al. [39] studied the photoelectron emission spectrum of $\mathrm{N}_{2}$ driven by the CRTCCP field, and found that the scattered electrons affect the spectrum. In 2019, Qiao et al. [40] systematically investigated the $\mathrm{HHG}$ of $\mathrm{H}_{2}^{+}$irradiated by the CRTCCP laser pulses. Their simulated results showed that at a certain field amplitude ratio, the harmonic efficiency is significantly improved and some harmonics have a circular polarization feature.

These studies mainly focus on the interaction of atoms with the laser pulses composed of the fundamental frequency and its second harmonic, while few research works focus on the laser pulses composed of the fundamental frequency and the higher frequency. HHG is usually considered a process which absorbs multiple photons and emits high-energy ones. For the interaction of the CRTCCP laser pulse with atoms, due to the angular momentum conservation, the absolute value of the angular momentum possessed by high-energy electrons that absorb multiple photons is greater than 1; as a result, it cannot directly jump to the ground state of the system to generate high-order harmonic. In this paper, we systematically studied the light emitted from the atom irradiated by the CRTCCP laser pulses composed of fundamental frequency and different times of the fundamental frequency. Obvious light emission can be observed under certain frequency conditions. By analyzing the polarization of the photons emitted, we found that these high-energy photons are generated by the transition from high-energy states to lower-energy states through multi-photon emission. On this basis, the relationship between the harmonics generated by this mechanism and parameters of the driving laser field is studied in detail.

To simulate the harmonic generation process of atom driven by CRTCCP laser pulses, the TDSE is numerically solved in this paper. The details of this scheme are given in Section 2. In Section 3, the harmonic spectrum of atom driven by CRTCCP is calculated, and the cause for its generation is explained from the perspective of quantum transition. We summarize the results in Section 4.

\section{Materials and Methods}

To calculate the harmonic spectrum of atoms irradiated by laser pulse, we need to solve the TDSE which describes the interaction between the laser electric field and electrons of atoms (atomic units are used throughout this paper, unless otherwise stated):

$$
i \frac{\partial}{\partial t} \varphi(\vec{r}, t)=\left[\frac{1}{2} \nabla^{2}+V(\vec{r})+\vec{E}(t) \cdot \vec{r}\right] \varphi(\vec{r}, t)
$$

where $\varphi(\overrightarrow{\boldsymbol{r}}, t)$ is the time-dependent spatial wave function of electrons. $V(r)=-k / \sqrt{|\overrightarrow{\boldsymbol{r}}|^{2}+a}$ is the attraction potential of the nucleus felt by the electrons, whose parameters are selected as $k=1$ a.u. and $a=0.393$ a.u., and the corresponding energy of ground state of Ar is 
-0.579 a.u. The third term in the bracket is the interaction between the laser electric field and the electrons. The driving laser we choose is a CRTCCP laser field, and the expression of $\vec{E}(t)$ is as follows:

$$
\begin{gathered}
\overrightarrow{\boldsymbol{E}}=E_{x}(t) \overrightarrow{\boldsymbol{e}}_{x}+E_{y}(t) \overrightarrow{\boldsymbol{e}}_{y} \\
E_{x}=f(t)\left[E_{1} \cos (\omega t)+E_{2} \cos (q \omega t)\right] \\
E_{y}=f(t)\left[E_{1} \sin (\omega t)+E_{2} \sin (q \omega t)\right]
\end{gathered}
$$

where $f(t)$ is the envelope of the laser field (a trapezoidal envelope with 8 optical cycles is used, and the rising and falling parts are both 1 optical cycle). $E_{1}$ and $E_{2}$ are the peak amplitude of the fundamental frequency field and higher-frequency field of the laser pulse, respectively. The fundamental frequency of the laser pulse is $\omega=0.057 \mathrm{a} . \mathrm{u}$. (corresponds to a central wavelength $\lambda=800 \mathrm{~nm}$ ), and the frequency of the high-frequency field is $q \omega$ ( $q$ is a positive integer). After obtaining the system wave function at arbitrary moment $\varphi(\vec{r}, t)$, the time-dependent dipole moment in the form of acceleration in the $x$ and $y$ directions can be calculated:

$$
\begin{aligned}
& a_{x}(t)=\left\langle\varphi(\overrightarrow{\boldsymbol{r}}, t)\left|-\frac{\partial V(\overrightarrow{\boldsymbol{r}})}{\partial x}-E_{x}(t)\right| \varphi(\overrightarrow{\boldsymbol{r}}, t)\right\rangle \\
& a_{y}(t)=\left\langle\varphi(\overrightarrow{\boldsymbol{r}}, t)\left|-\frac{\partial V(\overrightarrow{\boldsymbol{r}})}{\partial y}-E_{y}(t)\right| \varphi(\overrightarrow{\boldsymbol{r}}, t)\right\rangle
\end{aligned}
$$

The corresponding harmonic spectrum can be calculated by the Fourier transformation of the time-dependent dipole moment:

$$
\begin{aligned}
& P_{x}(\omega)=\left|\frac{1}{\omega^{2}\left(t_{n}-t_{0}\right)} \int_{t_{0}}^{t_{n}} a_{x}(t) e^{-i \omega t} \mathrm{~d} t\right|^{2} \\
& P_{y}(\omega)=\left|\frac{1}{\omega^{2}\left(t_{n}-t_{0}\right)} \int_{t_{0}}^{t_{n}} a_{y}(t) e^{-i \omega t} \mathrm{~d} t\right|^{2}
\end{aligned}
$$

In the formula, $t_{0}$ is the specified initial time, and $t_{n}$ is the time when the laser pulse ends. In order to understand the physical mechanism of HHG, the wavelet transform is used to analyze the time-frequency behavior of HHG:

$$
\begin{aligned}
& A_{x}\left(t_{0}, \omega\right)=\int_{t_{0}}^{t_{n}} a_{x}(t) w_{t_{0}, \omega}(t) \mathrm{d} t \\
& A_{y}\left(t_{0}, \omega\right)=\int_{t_{0}}^{t_{n}} a_{y}(t) w_{t_{0}, \omega}(t) \mathrm{d} t
\end{aligned}
$$

where $w_{t_{0}, \omega}(t)=\sqrt{\omega} W\left[\omega\left(t-t_{0}\right)\right]$ is the wavelet transform kernel, and the selection of the Morlet wavelet transform is as follows:

$$
W(s)=\frac{1}{\sqrt{\tau}} \mathrm{e}^{-i s} e^{-\frac{s^{2}}{2 \tau^{2}}}
$$

\section{Results and Discussion}

The peak amplitudes of the fundamental frequency field and the high-frequency field are chosen as $E_{1}=0.01$ a.u., $E_{2}=0.05$ a.u., respectively. Figure 1 a exhibits the harmonic spectrum from the Ar atom as a function of the high-frequency of the CRTCCP laser field. For comparison, we also calculate the harmonic spectrum when the polarization directions of the two circularly polarized lights in the combined pulse are opposite, as shown in Figure $1 \mathrm{~b}$. It can be seen from the figure that whether the driving field is a co-rotating two-color laser field or a counter-rotating two-color one, as $q$ increases, the radiation at other frequencies gradually disappears. When $q$ is greater than 12 , only the high frequency spectrum can be observed. It can be seen from the figure that there is an obvious light emission near $7 \omega$, which corresponds to the transition between the ground state and the excited state. When $q$ is lower than 12 , the harmonic spectrum has a relatively complicated 
distribution; especially for the high-frequency $q=5$, it can be observed that the intensity of its spectral emission is very large in many harmonic orders. For this reason, we conducted a detailed study on the harmonic generation process in the case of $q=5$.
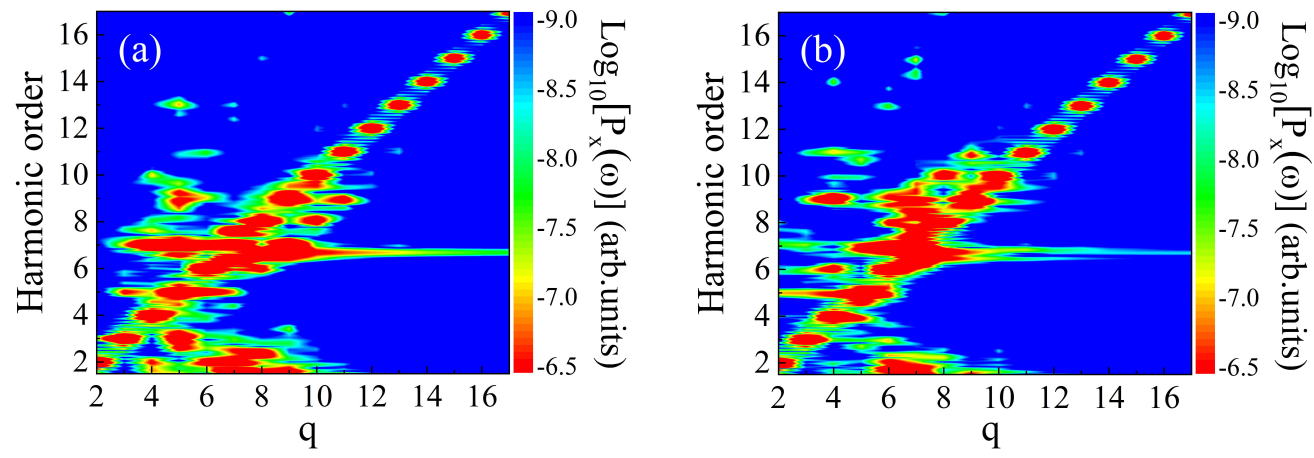

Figure 1. Harmonic spectrum of atom driven by the (a) co- and (b) counter-rotating two-color field as a function of the of the high frequency laser field. The electric field peak amplitudes of the fundamental frequency field and the high-frequency field are $E_{1}=0.01$ a.u. and $E_{2}=0.05$ a.u.

Figure 2 shows the harmonic spectrum of atom irradiated by the co-rotating and counter-rotating two-color laser pulses in the case of $q=5$. The 1st-, 5th-, 11th-, 13th-, 17thand 19th-order harmonics can be observed in the harmonic spectrum under the action of the counter-rotating two-color laser pulse. The generation of the 1st- and 5th-order harmonics here can be understood as the absorption of a low-energy photon and a highenergy photon, separately, and then photons are emitted when the electron jumps back to the ground state. Other harmonic generation corresponds to the process of emitting one photon after absorbing multiple photons. For example, the 11th-order harmonic is generated by absorbing two high-energy photons and one low-energy photon, and the 13th-order harmonic is generated by absorbing two high-energy photons and three lowenergy photons. In this process, the conservation of angular momentum is satisfied, i.e., the absolute value of the angular momentum of the photon emitted by the downward transition is 1 . It is worth noting here that under the action of the CRTCCP laser, not only can the HHG be observed in the system, but also the intensity of the HHG is significantly higher than that of the harmonics generated under the action of the counter-rotating two-color laser field.

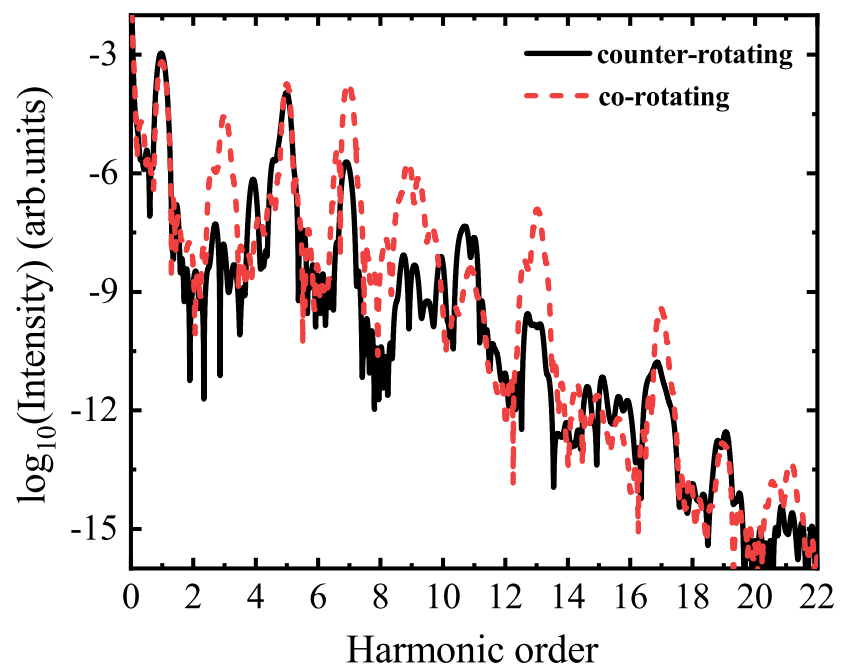

Figure 2. Harmonic spectrum of atom in the two-color field with $q=5, E_{1}=0.01$ a.u., $E_{2}=0.05$ a.u. The solid black and dashed red lines are the harmonic spectrum generated in counter- and co-rotating two-color field, separately. 
According to the model of absorbing multiple photons and emitting one photon, for the atom under the action of the co-rotating combined laser pulse, after absorbing several corotating circularly polarized photons, its angular momentum will be very large, and there is no way to jump to the ground state to produce high-energy photons. In order to understand the origin of this HHG, we use wavelet transform to study its time-frequency behavior.

Figure 3 shows the time-frequency behavior of the HHG of atoms driven by the CRTCCP laser pulse. Here, to analyze the HHG behavior of different polarization degrees, we combine the dipole moments of the two directions into $a_{x}+i a_{y}$ and $a_{x}-i a_{y}$, and the corresponding time-frequency behaviors are shown in Figure $3 a, b$, respectively. It can be seen from Figure 3 that for $a_{x}+i a_{y}$, the time-frequency behavior of its HHG can only observe the generation of the 3rd-and 7th-order harmonics, while for $a_{x}-i a_{y}$, the 5th-, 9thand 13th-order harmonics can be seen. It can be also seen from the figure that under the action of the CRTCCP laser, the light emission is not like the usual linearly polarized laser, which has a long and short emission trajectory in every half cycle. However, the behavior of HHG is close to that below the ionization threshold of linear polarization, i.e., the HHG can be observed during the entire laser pulse action time.
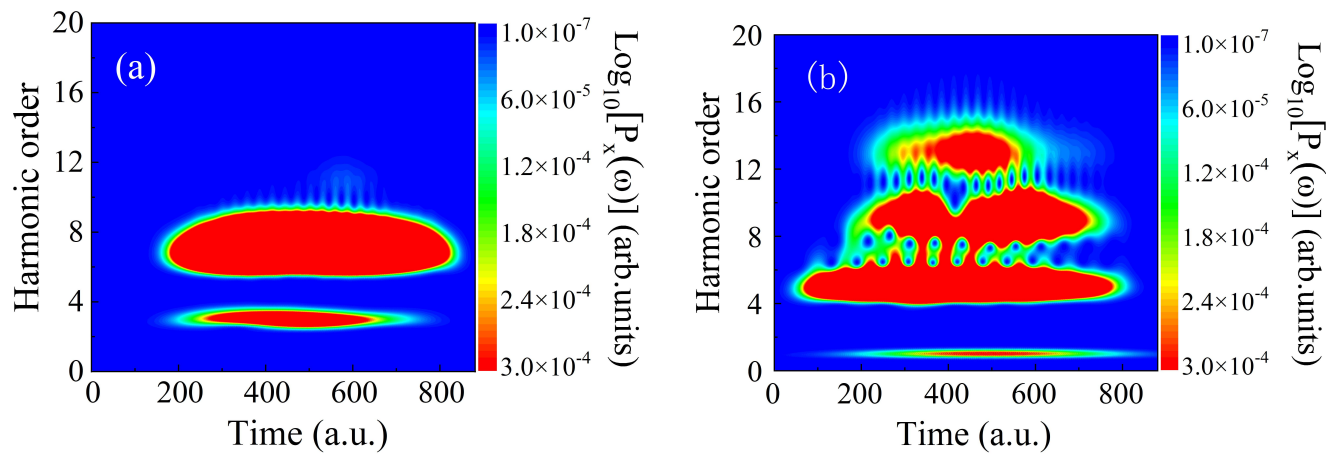

Figure 3. Wavelet analysis of the harmonics generated by the atoms in the CRTCCP field with $q=5$, $E_{1}=0.01$ a.u., $E_{2}=0.05$ a.u., (a) $a_{x}+i a_{y}$, (b) $a_{x}-i a_{y}$.

In order to understand this unique harmonic generation process, we propose a multiphoton absorption multi-photon radiation (MPA-MPR) model, as shown in Figure 4. Figure 4 a shows the harmonic spectrum of atom generated by the CRTCCP laser pulse with $\omega-5 \omega$, and Figure $4 \mathrm{~b}$ shows the schematic diagram of the quantum transition for the generation of 7th- and 9th-order harmonics. It can be seen from Figure 4 that according to the conservation of energy, the generation of the 7th-order harmonic needs to absorb two high-frequency photons, and emit three fundamental-frequency photons at the same time, and the generation of the 9th-order harmonic requires to absorb two high-frequency photons and radiate a fundamental frequency photon at the same time. Since the polarization of the high-frequency and fundamental fields of the driving laser are the same, the conservation of angular momentum can be used to determine that the rotation direction of the 7th-order harmonic should be opposite to the driven field, and the rotation direction of the 9th-order harmonic should be the same as the driving field. This model can also be used to explain the generation of higher order harmonics. The generation of the 11th- and 13th-order harmonics is achieved by the absorption of three high-energy photons. 


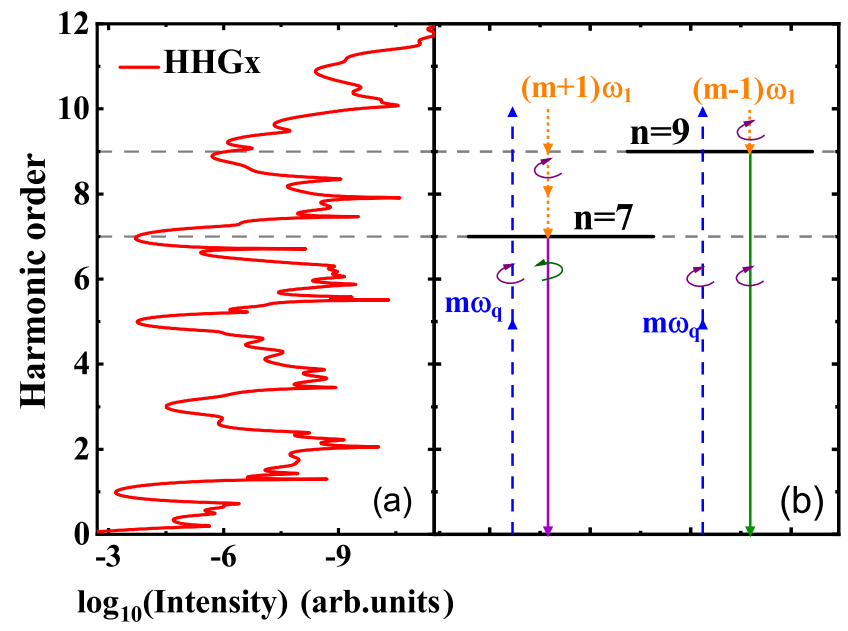

Figure 4. Harmonic spectrum (a) and their quantum transition diagram (b) of atom driven by the CRTCCP with $q=5, E_{1}=0.01$ a.u., $E_{2}=0.05$ a.u.

To verify the above speculation, we calculate the degree of polarization of the HHG, as shown in Figure 5. The formula for the degree of harmonic polarization is [41]

$$
\begin{gathered}
\varepsilon=\left[\left(\left|D_{+}\right|\right)-\left(\left|D_{-}\right|\right)\right] /\left[\left(\left|D_{+}\right|\right)+\left(\left|D_{-}\right|\right)\right] \\
D_{ \pm}=\frac{1}{\sqrt{2}}\left(D_{x} \pm i D_{y}\right)
\end{gathered}
$$

where $D_{x}$ and $D_{y}$ are the $x$ and $y$ components of the dipole moment in the frequency domain.

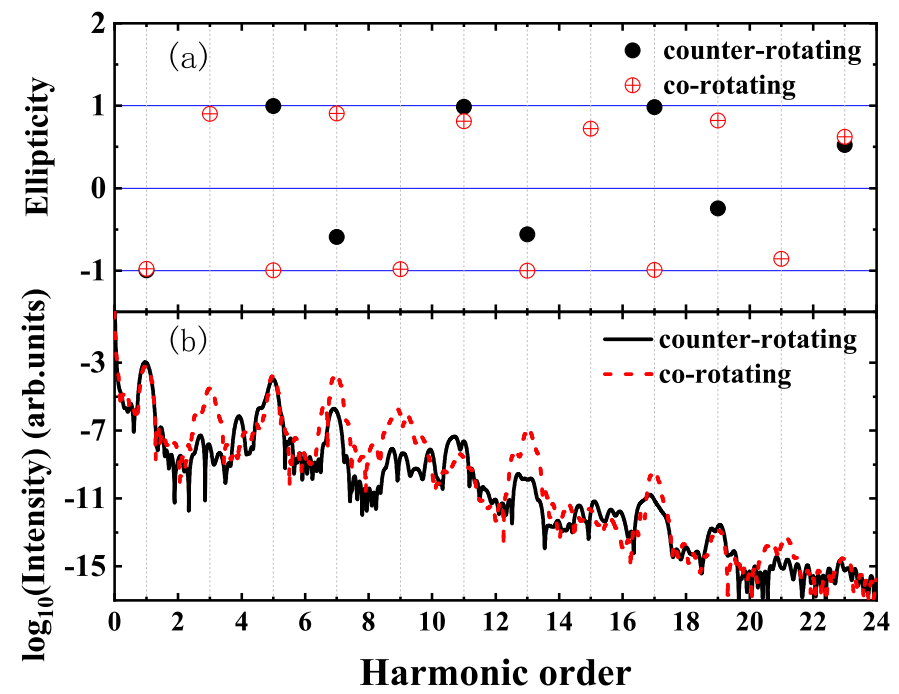

Figure 5. Harmonic spectrum (b) and the corresponding polarization of harmonics (a) generated by atoms when the driven field is co-rotating (red) and counter-rotating (black) two-color field with $q=5, E_{1}=0.01$ a.u., $E_{2}=0.05$ a.u.

The HHG and its polarization under the action of the counter-rotating two-color field are also shown in Figure 5. It can be seen from the figure that for the atom under the action of the counter-rotating two-color laser pulse, the generation of the 7th-order harmonic can be observed. According to the conservation of energy, to generate the 7th-order harmonic, the atom should absorb two low-frequency photons and one high-frequency photon to transition to a high energy level, and then jump from the high energy level to the ground state. The polarization of the 7th-order harmonic should be consistent with the polarization of the fundamental frequency field in the driving laser. On this basis, we observe that 
the degree of polarization of the 7th-order harmonic generated under the action of the co-rotating two-color field is opposite to the polarization of the driving laser pulse. The result is consistent with the theoretical prediction given above. Similarly, the polarization properties of the 9th-order harmonic are also consistent with the previous theory. It can be seen from the figure that the degree of polarization of the harmonic consistent with the polarization of the driving laser is very close to -1 , which means that circularly polarized harmonic pulses with a very high degree of polarization can be generated. Such pulses have important applications in the detection of magnetic materials, etc. For better applications, we need to optimize the harmonic efficiency. Usually, the field strength of the two-color field in the driving combined laser pulse has an important influence on the HHG. Next, the influence of this factor on the intensity of the HHG is studied.

We first fix the field strength of one frequency component in the two-color field, and study the influence of that of the other frequency one on the intensity of the harmonics, as shown in Figure 6. It can be seen from Figure 6a that when the peak amplitude of the high-frequency laser field in the driving laser pulse is 0.05 a.u., with the increase in the fundamental frequency field strength in the pulse, the generated harmonic intensity shows an overall decrease trend. Taking the 11th- and 13th-order harmonics as examples, when the amplitude of the fundamental frequency field is enhanced, it is observed that the intensity of the harmonics of the high-order increases and the intensity of the harmonics of the low-order decreases. It is believed that electrons are blown far away from the nucleus by the fundamental frequency field, increasing the probability of ionization.
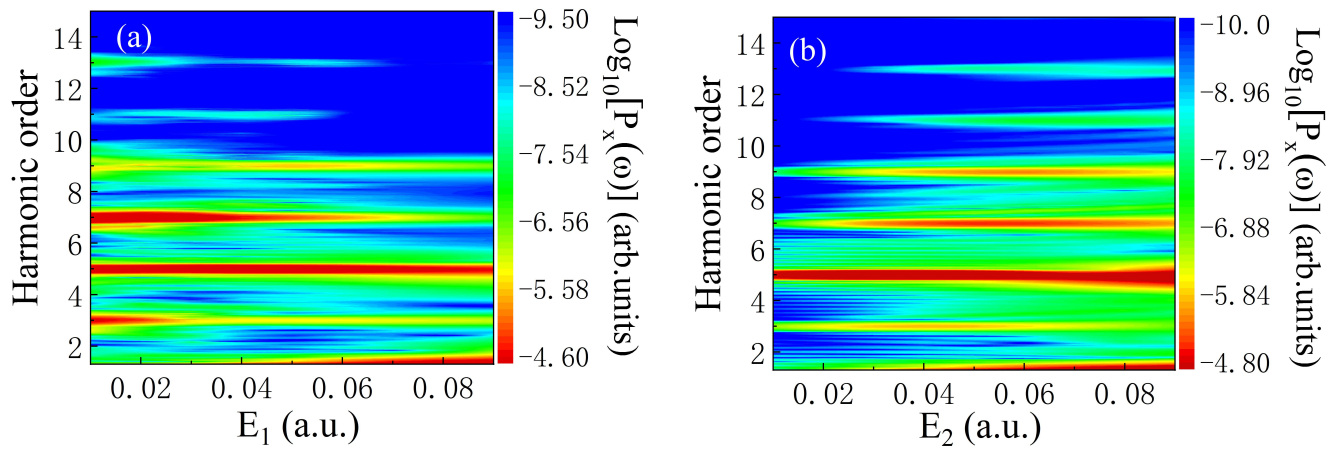

Figure 6. Harmonic intensity of atom as a function of the amplitude of the incident field when the incident field is a CRTCCP field with $q=5$. (a) shows the change of harmonic intensity with the amplitude of low-frequency field $E_{1}$, and $E_{2}=0.05$ a.u.; (b) shows the change of harmonic intensity with the amplitude of high-frequency field $E_{2}$, and $E_{1}=0.05$ a.u.

When the fundamental frequency field intensity is strong enough, no effective HHG can be observed.The intensities of the 11th- and 13th-order harmonics decrease as the intensity of the incident fundamental frequency field increases. In the CRTCCP field, the generation of 11th- and 13th-order harmonics just needs to absorb three high-energy photons. When the low-frequency field intensity of the driving laser is low, the electrons have absorbed three high-energy photons, ionized, and then recombined with the mother ion to generate harmonics. As the intensity of the fundamental frequency field increases, the part of ionized electron is derived away from the nucleus and cannot generate the harmonic emission. This phenomenon can be attributed to the fact that under the action of a high-frequency laser, the atoms can be effectively ionized. As the amplitude of the low-frequency laser electric field increases, the electrons are driven to a position that is far away from the nuclear region, and there is no chance for them to return to the ground state of the system to generate harmonics. Therefore, the intensity of these harmonics decreases as the amplitude of the fundamental frequency field increases. On this basis, we further studied the variation of the harmonic intensity with the amplitude of the highfrequency laser field when the peak amplitude of the low-frequency field in the driving laser pulse is 0.05 a.u., as shown in Figure 6b. From the figure, we can see that under 
this condition, as the high-frequency field strength increases, the intensity of the 11thand 13th-order harmonics gradually increases. The generation of 11th- and 13th-order harmonics requires the absorption of three high-energy photons. As the high frequency field strength increases, the probability of transition to a higher energy state increases, as does that of the downward transition, generating these harmonics. Therefore, by adjusting the amplitude of the CRTCCP field, the harmonic intensity can be optimized to generate a circularly polarized harmonic pulse with very high efficiency.

\section{Conclusions}

In summary, we theoretically study the harmonic generation process of atom in the CRTCCP laser pulse composed of fundamental-frequency and high-frequency fields of the same polarization direction. Compared with the harmonic generated by the combined laser pulse with the opposite polarization direction, it is found that the harmonic intensity generated by the combined laser pulse of the same polarization properties is higher. Further research shows that the mechanism of the HHG from atoms under the action of a laser pulse of the same polarization originates from the process of MPA-MPR. That is, driven by the CRTCCP laser pulse, the generation of harmonics requires absorbing multiple highfrequency photons, then generating the harmonics, and emitting multiple low-frequency photons. In addition, the influence of the intensity of the driving laser pulse on the harmonic is investigated. One can obtain a circularly polarized harmonic light source of higher intensity, changing the driving laser pulse intensity.

Author Contributions: Y.C.: data curation, writing-original draft, and software. X.S.: writing program. W.L.: editing. S.L.: editing. F.G.: supervision. Y.Y.: conceptualization, and supervision. All authors have read and agreed to the published version of the manuscript.

Funding: This research was funded by the National Key Research and Development Program of China (Grant No.2019YFA0307700), National Natural Science Foundation of China (NSFC) (Grant Nos. 12074145 and 11627807,11774129$)$.

Institutional Review Board Statement: Not applicable.

Informed Consent Statement: Not applicable.

Data Availability Statement: The data presented in this study are available on request from the corresponding author.

Acknowledgments: We acknowledge the High Performance Computing Center of Jilin University for the supercomputer time and the high performance computing cluster Tiger@ IAMP.

Conflicts of Interest: The authors declare no conflict of interest.

\section{References}

1. Agostini, P.; Fabre, F.; Mainfray, G.; Petite, G.; Rahman, N.K. Free-free transitions following six-photon ionization of xenon atoms. Phys. Rev. Lett. 1979, 42, 1127. [CrossRef]

2. Tian, Y.Y.; Li, S.Y.; Wei, S.S.; Guo, F.M.; Zeng, S.L.; Chen, J.G.; Yang, Y.J. Investigation on the influence of atomic potentials on the above threshold ionization. Chin. Phys. B 2014, 23, 053202. [CrossRef]

3. Shore, B.; Knight, P. Enhancement of high optical harmonics by excess-photon ionisation. J. Phys. B At. Mol. Phys. 1987, 20, 413. [CrossRef]

4. McPherson, A.; Gibson, G.; Jara, H.; Johann, U.; Luk, T.S.; McIntyre, I.; Boyer, K.; Rhodes, C.K. Studies of multiphoton production of vacuum-ultraviolet radiation in the rare gases. JOSA B 1987, 4, 595-601. [CrossRef]

5. Ferray, M.; L'Huillier, A.; Li, X.; Lompre, L.; Mainfray, G.; Manus, C. Multiple-harmonic conversion of $1064 \mathrm{~nm}$ radiation in rare gases. J. Phys. B At. Mol. Opt. Phys. 1988, 21, L31. [CrossRef]

6. Paul, P.M.; Toma, E.S.; Breger, P.; Mullot, G.; Augé, F.; Balcou, P.; Muller, H.G.; Agostini, P. Observation of a train of attosecond pulses from high harmonic generation. Science 2001, 292, 1689-1692. [CrossRef]

7. Ozaki, T.; Ganeev, R.; Ishizawa, A.; Kanai, T.; Kuroda, H. Highly directive $18.9 \mathrm{~nm}$ nickel-like molybdenum X-ray laser operating at $150 \mathrm{~mJ}$ pump energy. Phys. Rev. Lett. 2002, 89, 253902. [CrossRef]

8. Nabekawa, Y.; Hasegawa, H.; Takahashi, E.J.; Midorikawa, K. Production of doubly charged helium ions by two-photon absorption of an intense sub-10-fs soft X-ray pulse at $42 \mathrm{eV}$ photon energy. Phys. Rev. Lett. 2005, 94, 043001. [CrossRef] 
9. Pert, G. Optimizing the performance of nickel-like collisionally pumped X-ray lasers. II. Lasers for the wavelength range $50-100 \AA$. Phys. Rev. A 2007, 75, 023808. [CrossRef]

10. Shintake, T.; Tanaka, H.; Hara, T.; Tanaka, T.; Togawa, K.; Yabashi, M.; Otake, Y.; Asano, Y.; Bizen, T.; Fukui, T.; et al. A compact free-electron laser for generating coherent radiation in the extreme ultraviolet region. Nat. Photonics 2008, 2, 555-559. [CrossRef]

11. Popmintchev, T.; Chen, M.C.; Arpin, P.; Murnane, M.M.; Kapteyn, H.C. The attosecond nonlinear optics of bright coherent X-ray generation. Nat. Photonics 2010, 4, 822-832. [CrossRef]

12. Emma, P.; Akre, R.; Arthur, J.; Bionta, R.; Bostedt, C.; Bozek, J.; Brachmann, A.; Bucksbaum, P.; Coffee, R.; Decker, F.J.; et al. First lasing and operation of an ångstrom-wavelength free-electron laser. Nat. Photonics 2010, 4, 641-647. [CrossRef]

13. Hentschel, M.; Kienberger, R.; Spielmann, C.; Reider, G.A.; Milosevic, N.; Brabec, T.; Corkum, P.; Heinzmann, U.; Drescher, M.; Krausz, F. Attosecond metrology. Nature 2001, 414, 509-513. [CrossRef] [PubMed]

14. Sansone, G.; Benedetti, E.; Calegari, F.; Vozzi, C.; Avaldi, L.; Flammini, R.; Poletto, L.; Villoresi, P.; Altucci, C.; Velotta, R.; et al. Isolated single-cycle attosecond pulses. Science 2006, 314, 443-446. [CrossRef]

15. Guo, F.-M.; Yang, Y.-J.; Jin, M.-X.; Ding, D.-J.; Zhu, Q.-R. A theoretical strategy to generate an isolated 80-attosecond pulse. Chin. Phys. Lett. 2009, 26, 053201. [CrossRef]

16. Krausz, F.; Ivanov, M. Attosecond physics. Rev. Mod. Phys. 2009, 81, 163. [CrossRef]

17. Xia, C.-L.; Miao, X.-Y. Generation of linear isolated sub-60 attosecond pulses by combining a circularly polarized pulse with an elliptically polarized pulse. Chin. Phys. Lett. 2015, 32, 043202.

18. Wang, J.; Chen, G.; Li, S.-Y.; Ding, D.-J.; Chen, J.G.; Guo, F.-M.; Yang, Y.-J. Ultrashort-attosecond-pulse generation by reducing harmonic chirp with a spatially inhomogeneous electric field. Phys. Rev. A 2015, 92, 3.

19. van der Zwan, E.V.; Chirilă, C.C.; Lein, M. Molecular orbital tomography using short laser pulses. Phys. Rev. A 2008, 78, 033410. [CrossRef]

20. Wong, M.; Le, A.T.; Alharbi, A.; Boguslavskiy, A.; Lucchese, R.; Brichta, J.P.; Lin, C.; Bhardwaj, V. High harmonic spectroscopy of the cooper minimum in molecules. Phys. Rev. Lett. 2013, 110, 033006. [CrossRef]

21. Chen, J.; Yang, Y.; Chen, J.; Wang, B. Probing dynamic information and spatial structure of Rydberg wave packets by harmonic spectra in a few-cycle laser pulse. Phys. Rev. A 2015, 91, 043403. [CrossRef]

22. Corkum, P.B. Plasma perspective on strong field multiphoton ionization. Phys. Rev. Lett. 1993, 71, 1994. [CrossRef]

23. Yang, Y.J.; Chen, J.G.; Chi, F.P.; Zhu, Q.R.; Zhang, H.X.; Sun, J.Z. Ultrahigh harmonic generation from an atom with superposition of ground state and highly excited states. Chin. Phys. Lett. 2007, 24, 1540.

24. de Morisson Faria, C.F.; Dörr, M.; Sandner, W. Importance of excited bound states in harmonic generation. Phys. Rev. A 1998, 58, 2990. [CrossRef]

25. Kfir, O.; Grychtol, P.; Turgut, E.; Knut, R.; Zusin, D.; Popmintchev, D.; Popmintchev, T.; Nembach, H.; Shaw, J.M.; Fleischer, A.; et al. Generation of bright phase-matched circularly-polarized extreme ultraviolet high harmonics. Nat. Photonics 2015, 9, 99-105. [CrossRef]

26. Miao, X.Y.; Du, H.N. Theoretical study of high-order-harmonic generation from asymmetric diatomic molecules. Phys. Rev. A 2013, 87, 053403. [CrossRef]

27. Miao, X.Y.; Zhang, C.P. Multichannel recombination in high-order-harmonic generation from asymmetric molecular ions. Phys. Rev. A 2014, 89, 033410. [CrossRef]

28. Reich, D.M.; Madsen, L.B. Illuminating molecular symmetries with bicircular high-order-harmonic generation. Phys. Rev. Lett. 2016, 117, 133902. [CrossRef]

29. Baykusheva, D.; Ahsan, M.S.; Lin, N.; Wörner, H.J. Bicircular high-harmonic spectroscopy reveals dynamical symmetries of atoms and molecules. Phys. Rev. Lett. 2016, 116, 123001. [CrossRef]

30. Wang, J.; Chen, G.; Guo, F.M.; Li, S.Y.; Chen, J.G.; Yang, Y.J. High-intensity molecular harmonic generation without ionization. Chin. Phys. B 2013, 22, 033203. [CrossRef]

31. Neufeld, O.; Ayuso, D.; Decleva, P.; Ivanov, M.; Smirnova, O.; Cohen, O. Ultrafast All-Optical Detection of Chiral Degrees of Freedom by Symmetry Breaking High Harmonic Spectroscopy. In Proceedings of the Nonlinear Optics 2019, Waikoloa Beach, HI, USA, 15-19 July 2019; p. NM1A-2.

32. Budil, K.; Salières, P.; L'Huillier, A.; Ditmire, T.; Perry, M. Influence of ellipticity on harmonic generation. Phys. Rev. A 1993, 48, R3437. [CrossRef] [PubMed]

33. Möller, M.; Cheng, Y.; Khan, S.D.; Zhao, B.; Zhao, K.; Chini, M.; Paulus, G.G.; Chang, Z. Dependence of high-order-harmonicgeneration yield on driving-laser ellipticity. Phys. Rev. A 2012, 86, 011401. [CrossRef]

34. Odžak, S.; Hasović, E.; Milošević, D.B. High-order harmonic generation in polyatomic molecules induced by a bicircular laser field. Phys. Rev. A 2016, 94, 033419. [CrossRef]

35. Jiménez-Galán, Á.; Zhavoronkov, N.; Schloz, M.; Morales, F.; Ivanov, M. Time-resolved high harmonic spectroscopy of dynamical symmetry breaking in bi-circular laser fields: The role of Rydberg states. Opt. Express 2017, 25, 22880-22896. [CrossRef] [PubMed]

36. Zhang, X.; Zhu, X.; Liu, X.; Wang, D.; Zhang, Q.; Lan, P.; Lu, P. Ellipticity-tunable attosecond XUV pulse generation with a rotating bichromatic circularly polarized laser field. Opt. Lett. 2017, 42, 1027-1030. [CrossRef] [PubMed]

37. Eichmann, H.; Egbert, A.; Nolte, S.; Momma, C.; Wellegehausen, B.; Becker, W.; Long, S.; McIver, J. Polarization-dependent high-order two-color mixing. Phys. Rev. A 1995, 51, R3414. [CrossRef]

38. Long, S.; Becker, W.; McIver, J. Model calculations of polarization-dependent two-color high-harmonic generation. Phys. Rev. A 1995, 52, 2262. [CrossRef] 
39. Busuladžić, M.; Čerkić, A.; Gazibegović-Busuladžić, A.; Hasović, E.; Milošević, D. Molecular-orientation-dependent interference and plateau structures in strong-field ionization of a diatomic molecule by a corotating bichromatic elliptically polarized laser field. Phys. Rev. A 2018, 98, 013413. [CrossRef]

40. Qiao, Y.; Wu, D.; Chen, J.G.; Wang, J.; Guo, F.M.; Yang, Y.J. High-order harmonic generation from $\mathrm{H}^{2+}$ irradiated by a co-rotating two-color circularly polarized laser field. Phys. Rev. A 2019, 100, 063428. [CrossRef]

41. Odžak, S.; Milošević, D. Bicircular-laser-field-assisted electron-ion radiative recombination. Phys. Rev. A 2015, $92,053416$. [CrossRef] 